\title{
Lumbo-costo-vertebral syndrome with posterior spinal dysraphism
}

\author{
G. Samson Sujit Kumar, Vaijayantee Kulkarni, R. P. Haran \\ Department of Neurological Sciences, Christian Medical College and Hospital, Vellore, India
}

\begin{abstract}
Lumbo-costo-vertebral syndrome, which includes abnormalities of the vertebral bodies, ribs and trunk musculature, is very rare and only few cases have been reported. We report a case of 18-month-old female child with absent ribs, hemivertebrae, superior lumbar hernia (features of lumbocosto-vertebral syndrome) and posterior spinal dysraphism, which to our knowledge is the first case in the English literature with such a combination of defects. Embryology and management of the case is discussed.
\end{abstract}

Key words: lumbar hernia; lumbo-costo-vertebral syndrome; myelomeningocoele; tethered cord.

Lumbo-costo-vertebral syndrome is a rare disorder in children. Only 12 cases have been reported in the literature so far. It includes hemivertebrae, congenital absence of ribs, anterior myelomeningocele and hypoplasia of the anterior abdominal wall presenting as congenital lumbar hernia. ${ }^{[1-4]}$ Cases of lumbo-costo-vertebral syndrome with other combination of defects have also been reported. We report a case of lumbocosto-vertebral syndrome (left superior lumbar hernia, hemivertebrae and absent ribs on the left side) associated with posterior spinal dysraphism and discuss the relevant embryology and role of multidisciplinary involvement in the management of these cases.

\section{Case report}

An 18-month-old female baby presented with history of swellings in the left hypochondrium and low back regions and straining while passing urine and stools since her birth. On examination, she was found to have a $10 \mathrm{~cm}$ reducible swelling in the left hypochondrium with an expansile cough impulse [Figure 1]. There was another swelling in the low back region, which was $4 \mathrm{~cm}$ ill-defined, soft irreducible and without a cough impulse. There was thoraco-lumbar scoliosis with convexity to the left [Figure 2]. The neurological examination of

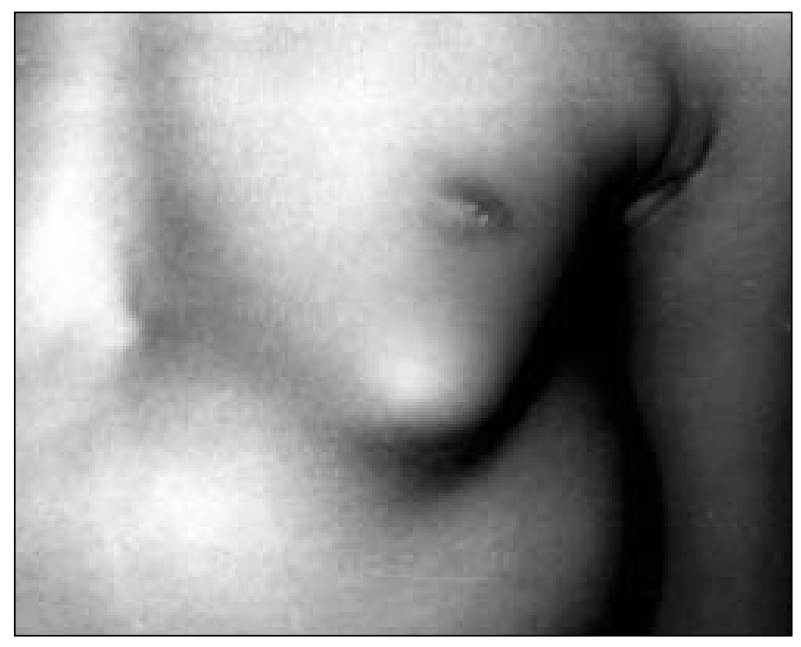

Figure 1: Clinical picture of the patient showing the left superior lumbar hernia

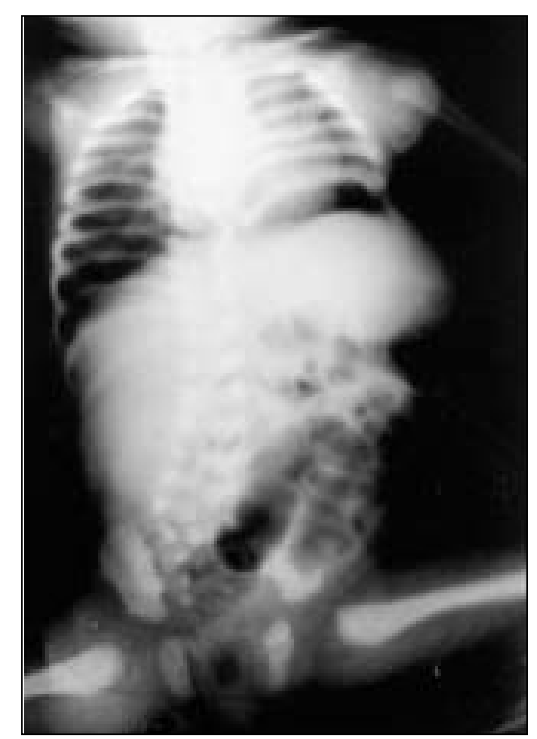

Figure 2: Plain X-rays of the thoracolumbar spine showing T7-T11 and S1 hemivertebra with absent left T7-T10ribs, hypoplastic left T11-T12 ribs and scoliosis 
the lower limbs was normal. Anal tone was normal and superficial anal reflex was elicitable.

Radiological investigations revealed T7 T11 and S1 hemivertebrae with absent left T7 T10 ribs and hypoplastic left T11 T12 ribs [Figure 2]. Ultrasound abdomen and cystometrogram done were normal. Magnetic resonance imaging of the spine showed kyphoscoliosis of the thoracolumbar spine with hemivertebrae in the mid-thoracic region. Spinal cord was low lying, tethered at L4 and cord substance extending below L4. Lower lumbar and sacral dysraphism was noted with an intraspinal mass (L4-5 level), which was hyperintense on T1W and T2W sequences suggestive of a dorsal lipoma. Linear cavity was seen within the cord from L1-L4 vertebral levels suggestive of syringomyelia [Figures 3-5]. She underwent lumbar exploration, L4 laminectomy,

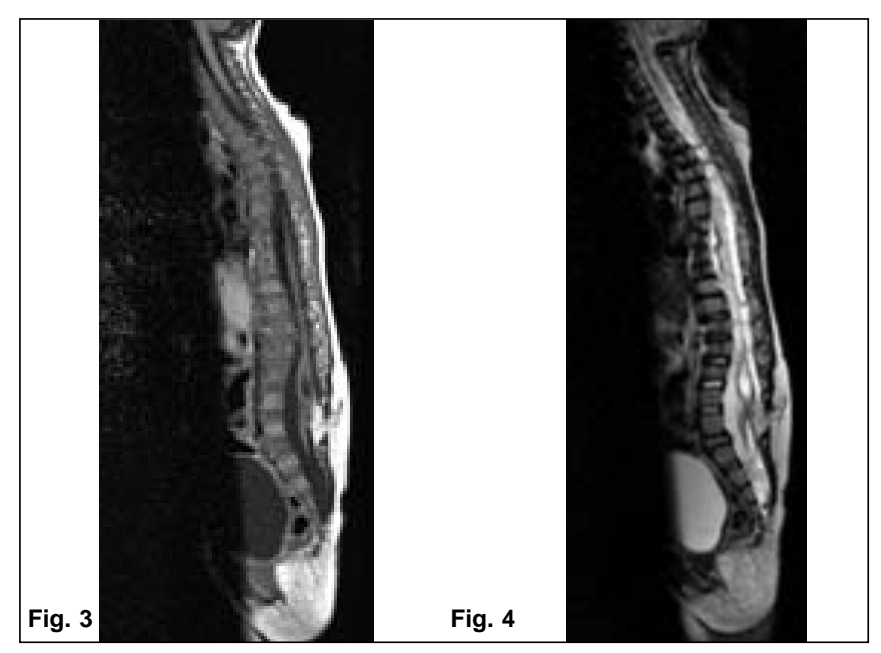

Figure $3 \&$ 4: T1W plain sagittal image of the spine showing the low lying cord, tethered at L4 with a hyperintense intraspinal lesion attached to the cord dorsally and extending into the subcutaneous fat through the spina bifida. There is a hypointense lesion within the cord above the level of tethering. Figure 4: T2W sagiital image shows that the intraspinal lesion is hyperintense(suggestive of lipoma) and the lesion within the spinal cord is hyperintense(suggestive of syrinx)

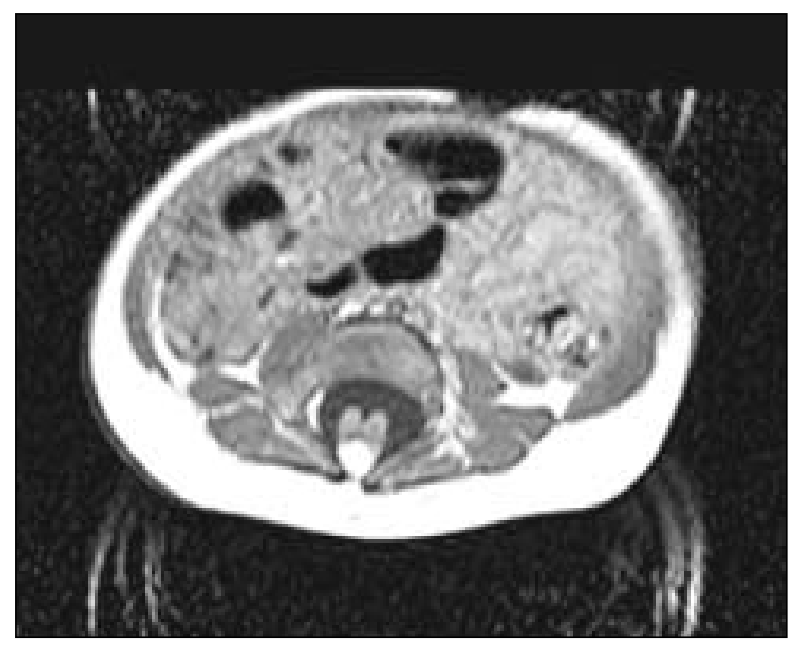

Figure 5: T1W axial images show that the hyperintense lesion is attached to the cord dorsally excision of subcutaneous and dorsal lipoma and untethering of the cord. At surgery there were two lipomas seen in the subcutaneous plane and they were adherent through the spina bifida at L4 level to the dorsal surface of the cord. These lipomas were released from all around and shaved off the cord. In the post-operative period she did not have any neurological deficit. She was followed up by the pediatric surgeons for the lumbar hernia and orthopaedicians for the scoliosis. After 7 years of follow up, she did not develop any new neurological deficit. There was no worsening of the scoliosis and she was asymptomatic for the lumbar hernia

\section{Discussion}

Lumbo-costo-vertebral syndrome includes hemi-vertebra, congenital absence of ribs, anterior myelomeningocoele_and abdominal wall muscular hypoplasia. These defects could probably due to a single somatic defect occurring during $3-5$ weeks of gestational age, which results in malformation of the vertebral bodies, ribs and abdominal musculature. ${ }^{[3]}$ Between 3 and 5 weeks of gestational age when the crown rump length of the embryo is $1.5 \mathrm{~mm}$, the mesoderm between the endoderm and ectoderm on either sides of the notochord differentiates into somites which further differentiate into the sclerotome that forms the vertebrae and costal process, the myotome that forms the skeletal muscles of the trunk and the dermatome which contributes to the deep layers of the skin and the subcutaneous tissue. ${ }^{[5]}$ Any disturbance during this stage, probably anoxia as demonstrated in animal studies will result in vertebral, costal and abdominal defects. ${ }^{[6]}$

During the 3-5 weeks of gestational age neurulation occurs during which formation of the neural groove, closure of the anterior (25 days) and posterior (27 to 28 days) neuropores, appearance of the ventral horn cells, formation of anterior and posterior roots and disjunction of the neuroectoderm from the surface ectoderm takes place. ${ }^{[5]}$ So the defects of the lumbo-costo-vertebral syndrome could be associated with the spinal cord malformations. In our case the patient had left superior lumbar hernia, hemivertebrae with absent left ribs and posterior spinal dysraphism with a dorsal lipoma and tethered cord at L4 level, which is a disorder of primary neurulation.

A lumbar hernia is a parietal wall defect that may occur anywhere in the lumbar region between the 12th rib and the iliac crest. Congenital lumbar hernia is rare. It can occur in the superior (Grynfelt Lesshaft's) or the inferior lumbar (Petit's) triangle. ${ }^{[7,8]}$ Previously published articles suggest an association between the lumbar hernias and lumbo-costo-vertebral syndrome defects. ${ }^{[3,4,9]}$ Other abnormalities associated with congenital lumbar hernia, reported so far include congenital sciatic hernia, absent tibia, posterior myelomeningocoele, focal nodular hyperplasia of the liver and absent kidney ${ }^{[10-12]}$ In the case of posterior myelomeningocoele association was more like a acquired lumbar hernia as it appeared after the repair 
of the myelomeningocoele

Our case describes a rare combination of hemivertebrae with absent ribs, left superior lumbar hernia and posterior spinal dysraphism with a dorsal lipoma and tethered cord. Untethering of the cord was done to prevent neurological complications that could arise. Now she is on regular follow up to monitor her neurological status, lumbar hernia and scoliosis. This case highlights the fact that it is vital to investigate these patients with X-ray abdomen, chest, ultrasound abdomen and magnetic resonance imaging of the spine to rule out the associated abnormalities and the need for regular multidisciplinary follow up.

\section{Reference}

1. Lee CM Jr, Mattheis H. Congenital lumbar hernia. Arch Dis Child $1957 ; 32: 42-7$.
2. Talukder BC.Congenital lumbar hernia. J Pediatr Surg 1974;9:419-20.

3. Touloukian RJ. The lumbocostovertebral syndrome: A single somatic defect. Surgery 1972;71:174-81.

4. Bernay F, Gidenex C, Gurses N. Superior lumbar hernia associated with the lumbocostovertebral syndrome. Pediatr Surg Int 1990;5:469-70.

5. Pang D. Spinal Cord Lipomas In Disorders of Pediatric spine,(ed) Raven press, Ltd: New York;1995. p. 178-9.

6. Ingalls TH, Curley F.J, Prendle RA. Experimental production of congenital anomalies: Timing and degree of anoxia as a factor causing fetal deaths and congenital anomalies in the mouse. N Engl J Med 1952;247:758.

7. Gyrnfelt J. Quelques mots sur la hernie lombaire. Montpellier Med 1866;16:329.

8. Lesshaft P. Die Lumbalgegend in Anatomische chirurgischer Hinsicht. Arch Anat Physiol. Wissensch Med 1870;37:264.

9. Hancock B.J, Wiseman NE. Incarcerated congenital lumbar hernia associated with the lumbocostovertebral syndrome. J Pediatr Surg 1988;23:782-3.

10. Loftus BG, O'Carroll T. Lumbar herniation and tibial absence in infants of diabetic mothers. Diabet Med 1985;2:283-5.

11. Lowell DG, Guzetta PC. Lumbar hernia in a case of posterior meningomyelocele. J Pediatr Surg 1986;21:913-4

12. al-Salem AH, Abu-Srair H, Qaissaruddin S. Focal nodular hyperplasia of the liver with the lumbo-costovertebral svndrome. .J Pediatr Surg 1996;31:1282-4.

Accepted on 29-08-2005 